\title{
DIVERSIDAD DE COLEOPTEROS ACUATICOS EN CHILE
}

\section{DIVERSITY OF FRESHWATER BEETLE OF CHILE}

\author{
Viviane Jerez $^{1}$ \& Juan Moroni ${ }^{2}$ \\ ${ }^{1}$ Departamento de Zoología. Facultad de Ciencias Naturales y Oceanográficas. Universidad de Concepción. \\ Casilla 160-C, Concepción, Chile. E-mail: vijerez@udec.cl \\ ${ }^{2}$ Servicio Agrícola y Ganadero, Oficina Comercio Exterior. \\ Aeropuerto C. A. Merino Benítez, Pudahuel, Santiago, Chile. E-mail: juan.moroni@sag.gob.cl
}

\section{RESUMEN}

\begin{abstract}
Se entrega para los coleópteros acuáticos, una reseña acerca de la diversidad y riqueza de familias, géneros y especies de los subórdenes Hydradephaga y Polyphaga, presentes en Chile. Hydradephaga está representado por las familias Dytiscidae, Gyrinidae y Haliplidae. Polyphaga por Hydrophilidae, Hydraenidae, Elmidae y Psephenidae. A excepción de Lancetes, que es el género más diversificado con 14 especies, la mayoría de los taxa de ambos subórdenes son monoespecíficos. En relación a aspectos biogeográficos, Chile no presenta familias endémicas. Sin embargo, se han descrito situaciones de endemismo de algunas especies para el Archipiélago de Juan Fernández (Lancetes backstroni, Anisomeria bistriata), e Isla de Pascua (Bidessus skottsbergi). En el continente existen elementos de origen tropical y australiano, como es el caso de Tropisternus y Austrolimnius de distribución neotropical, y Lancetes relacionado con fauna de Australia, Nueva Zelanda y Tasmania. En el trabajo se entrega además aspectos ecológicos de las familias y su potencial como bioindicadores.
\end{abstract}

Palabras claves: Dytiscidae, Gyrinidae Haliplidae, Hydrophilidae, Hydraenidae, Elmidae, Psephenidae, Chile.

ABSTRACT

For water beetles of Hydradephaga and Polyphaga suborders, the diversity of family, genera and species richness present in Chile is analyzed. Hydradephaga its represented by Dytiscidae, Gyrinidae and Haliplidae families and the Polyphaga by Hydrophilidae, Hydraenidae, Elmidae and Psephenidae. Exceptionally, Lancetes is the genus most diversified with 14 species. The most of taxa of both suborders are monospecific. In relation to biogeographic aspects, Chile does not present endemic families. Nevertheless endemism situation to species level has been described for the Archipiélago de Juan Fernández (Lancetes backstroni, Anisomeria bistriata), and Isla de Pascua (Bidessus skottsbergi). In the continent, elements of tropical and Australian origin exist; as it is the case of Tropisternus and Austrolimnius of neotropical distribution and Lancetes with Australian, New Zealand and Tasmania relations. In this work, ecological aspects of the families and their use as bioindicators are given.

Keywords: Dytiscidae, Gyrinidae Haliplidae, Hydrophilidae, Hydraenidae, Elmidae, Psephenidae, Chile. 


\section{INTRODUCCION}

La diversidad biológica y ecológica de los coleópteros es extraordinaria. Taxonómicamente constituyen más del $40 \%$ de la diversidad descrita para Hexapoda a nivel mundial, con aproximadamente 350.000 especies descritas a la fecha (Arias 2000). Para Chile, los coleópteros representan más del 90\% de la diversidad entomológica descrita, reconociéndose hasta el momento 96 familias y aproximadamente 1.196 géneros y 3.947 especies (Lawrence \& Newton 1995, Elgueta 2000).

Su gran diversidad ecológica se expresa en la capacidad de colonizar todo tipo de ambientes entre ellos el acuático, abarcando desde aguas continentales hasta sectores litorales o de marismas, y desde zonas litorales hasta ríos de alta montaña, e inclusive lagunas hipersalinas del norte grande. La evolución hacia los ambientes acuáticos pasa por múltiples adaptaciones, que incluyen aspectos morfológicos, fisiológicos y ontogenéticos y todas las familias adaptadas a vivir en estos ambientes presentan al menos, uno de sus estados de desarrollo en el agua. Por ejemplo, ditíscidos e hidrofílidos, que son las familias más frecuentes, presentan larvas y adultos totalmente acuáticos. Otras en cambio, como los élmidos y la mayoría de los hidraénidos, presentan fases larvarias acuáticas y adultos con fases mixtas (Trémouilles et al. 1995).

Todos los cuerpos de aguas continentales constituyen hábitats favorables para los coleópteros acuáticos donde forman parte de las comunidades lóticas (ritrón y potamón) y lénticas (Trémouilles et al. 1995). Ecológicamente, por lo tanto, estos insectos y principalmente su estado larvario forman parte de la fauna de macroinvertebrados, bentónicos participando en múltiples cadenas tróficas donde actúan como depredadores, detritívoros, herbívoros o comedores de perifiton. También actúan como eslabones tróficos intermedios entre productores primarios y consumidores secundarios (principalmente peces). Finalmente, estos insectos pueden actuar como transformadores e integradores de materia orgánica alóctona (i.e. hojas, semillas, ramas, troncos caídos) (Araya 2000).

Sin embargo, y a pesar de presentar una cierta uniformidad ecológica, desde un punto de vista taxonómico, los coleópteros acuáticos constituyen un grupo heterogéneo que incluye taxa pertenecientes a distintos linajes de los Subórdenes
Adephaga y Polyphaga. A nivel mundial es en los Adephaga donde se puede evidenciar más claramente la evolución hacia el ambiente acuático, con una radiación adaptativa y diversificación en seis familias: Dytiscidae, Gyrinidae, Haliplidae, Hygrobiidae y Amphizoidae. Todas estas familias están incluidas actualmente en la categoría Hydradephaga, y cuyo conjunto constituiría una unidad monofilética según recientes estudios moleculares (Ribera et al. 2002). La evolución y adaptación de los Hydradephaga hacia el ambiente acuático se expresa en cambios morfológicos de las patas y ornamentación del tegumento (crestas, carenas, gránulos, cerdas), destinados a la captación del oxígeno atmosférico. Estas adaptaciones del punto de vista ecológico les ha permitido utilizar todas las modalidades de vida acuática: agua superficial, sustratos flotantes, sumergidos entre dos aguas o de fondo, ambientes intersticiales y aguas freáticas. Para los que llevan a cabo la mayor parte de su desarrollo en el agua y solamente el estado de pupa es terrestre, se han descrito aproximadamente 5.000 especies a nivel mundial, distribuidas en 200 géneros. La familia más grande es Dytiscidae con 3.500 especies; le sigue Gyrinidae con 1.000, Noteridae con 270, Haliplidae con 220 e Hygrobiidae y Amphizoidae con seis especies, respectivamente. En Chile sólo están presentes Dytiscidae, Gyrinidae y Haliplidae. Los representantes del Suborden Polyphaga constituyen por el contrario, un grupo heterogéneo ya que las familias que se han adaptado a vivir en ambientes acuáticos pertenecen a distintos linajes. En Chile sólo están presentes cuatro familias, cuyos estados larvarios transcurren en el agua: Hydrophilidae, Hydraenidae, Elmidae y Psephenidae (Tabla I).

\section{DIVERSIDAD DE FAMILIAS Y ESPECIES}

En Chile están presentes tres familias de Hydradephaga, de las cuales Dytiscidae es la que presenta la mayor riqueza a nivel genérico y específico, con 11 géneros y 34 especies. Gyrinidae, en cambio, está representada por dos géneros y cuatro especies, y Haliplidae con un género y tres especies.

La Tabla II muestra la composición taxonómica de familias, subfamilias, géneros y número de especies descritas en Chile para Hydradephaga y Polyphaga. Se observa que entre los géneros de Dytiscidae, 
Gayana 70(1), 2006

TABLA I. Número de géneros y especies descritas para las familias de coleópteros dulceacuícolas chilenos.

TABLE I. Number of genera and species described for the Chilean freshwater coleoptera.

\begin{tabular}{llcc}
\hline Subórdenes & Familias & Géneros & Especies \\
\hline \multirow{2}{*}{ Adephaga } & Dytiscidae & 11 & 34 \\
& Gyrinidae & 2 & 4 \\
& Haliplidae & 1 & 3 \\
Polyphaga & Hydrophilidae & 13 & 26 \\
& Hydraenidae & 3 & 16 \\
& Elmidae & 7 & 16 \\
& Psephenidae & 3 & 3 \\
\hline Total & & 40 & 102 \\
\hline
\end{tabular}

Lancetes es el más diversificado con un total de 14 especies (ver Elgueta \& Arriagada 1989), seguido por Rhantus y Liodessus con cuatro especies cada uno (Moroni 1973, 1988). Los restantes géneros son monoespecíficos, salvo Laccophilus, Megadytes y Leuronectes, cada uno con dos especies. Los Polyphaga están representados en Chile por cuatro familias, de las cuales Hydrophilidae es la más diversificada con 13 géneros y 26 especies. La familia Elmidae presenta siete géneros destacando Austrelmis con ocho especies y Austrolimnius con dos especies. Hydraenidae presenta sólo tres géneros, de los cuales Gymnochithebius es el más diversificado con siete especies descritas. Finalmente, Psephenidae es la familia menos diversificada con tres géneros monoespecíficos.

Hasta la fecha sólo ha sido detectada una especie de Hydrophilidae, Sphaeridiinae, Dactylosternum abdominale, ampliamente distribuida en América Central, Estados Unidos y Región Neotropical.

\section{RESEÑA HISTÓRICA}

La primera descripción de un coleóptero acuático para Chile corresponde a Fabricius (1775), quien describe a Dytiscus signatus (=Rhantus). Posteriormente, se publicaron en Inglaterra, Francia y Alemania numerosas descripciones de coleópteros acuáticos recolectados en nuestro país, por científicos de diversas expediciones, entre los que se pueden citar a D'Orbigny, Fitzroy, King, Dumont D'Urville y otros. Todas las contribuciones sobre coleópteros acuáticos realizadas entre la publicación de Fabricius y la obra de Gay (1849) están resumidas en el catálogo de los Coleópteros de Chile (Philippi, 1887), en el cual Dytiscidae aparece representada por 12 géneros y 29 especies; Gyrinidae con dos géneros y cinco especies e Hydrophilidae con cuatro géneros y diez especies. Posteriormente, Germain (1911) en su "Catálogo de los coleópteros chilenos del Museo Nacional", sinonimiza varias especies descritas principalmente por autores europeos y también por Philippi (1864). En resumen, hasta la publicación de Germain, la diversidad de coleópteros acuáticos chilenos incluía a Dytiscidae con seis géneros y 12 especies, Gyrinidae con un género y dos especies, y finalmente Hydrophilidae con siete géneros y 14 especies. Desde Germain hasta fines de los 60, en que Moroni comenzó el estudio de estos grupos, basado en material conservado en la colección del Museo Nacional de Historia Natural, prácticamente nadie en Chile se había preocupado de incrementar el conocimiento de los coleópteros acuáticos presentes en el país. Desde entonces y gracias al interés de especialistas nacionales y extranjeros y al hecho de haberse efectuado diversas expediciones de reconocimiento de la entomofauna nacional, se ha más que duplicado el conocimiento de la diversidad de estos insectos en Chile.

\section{CONSIDERACIONES BIOGEOGRÁFICAS}

Chile no presenta familias endémicas de coleópteros acuáticos, a diferencia de otras regiones mediterráneas. Sin embargo, esta fauna muestra elementos sudamericanos de origen tropical y australiano. Es el caso de los géneros Tropisternus, que está ampliamente distribuido en la región neotropical y Lancetes que presenta nexos con Australia, Nueva Zelanda y Tasmania, al igual que el género de Psephenidae Tychepsephus (Lawrence \& Britton 1994) y Austrolimnius, taxa descrito como el género dominante de élmidos en aguas dulces, y que se encuentra también en América Central y del Sur (Hinton 1965). Por otra parte, la mayor parte de los géneros están poco diversificados y muchos de ellos son monotípicos, situación relacionada principalmente con el aislamiento del territorio desde el Terciario, con el levantamiento de la Cordillera de los Andes (Solervicens 1995). 
Coleópteros dulceacuícolas de Chile: Jerez, V. \& J. Moroni

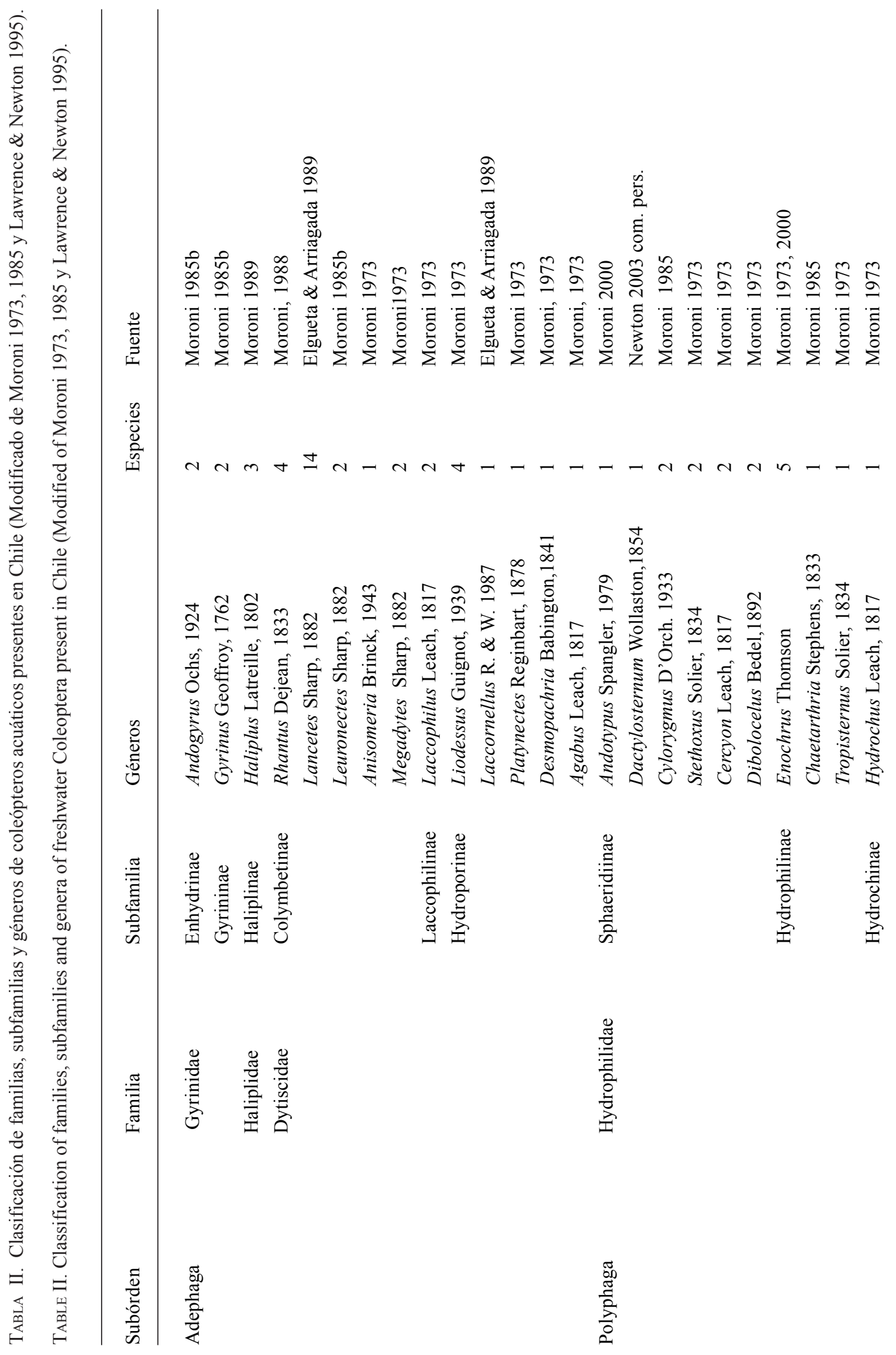


Gayana 70(1), 2006

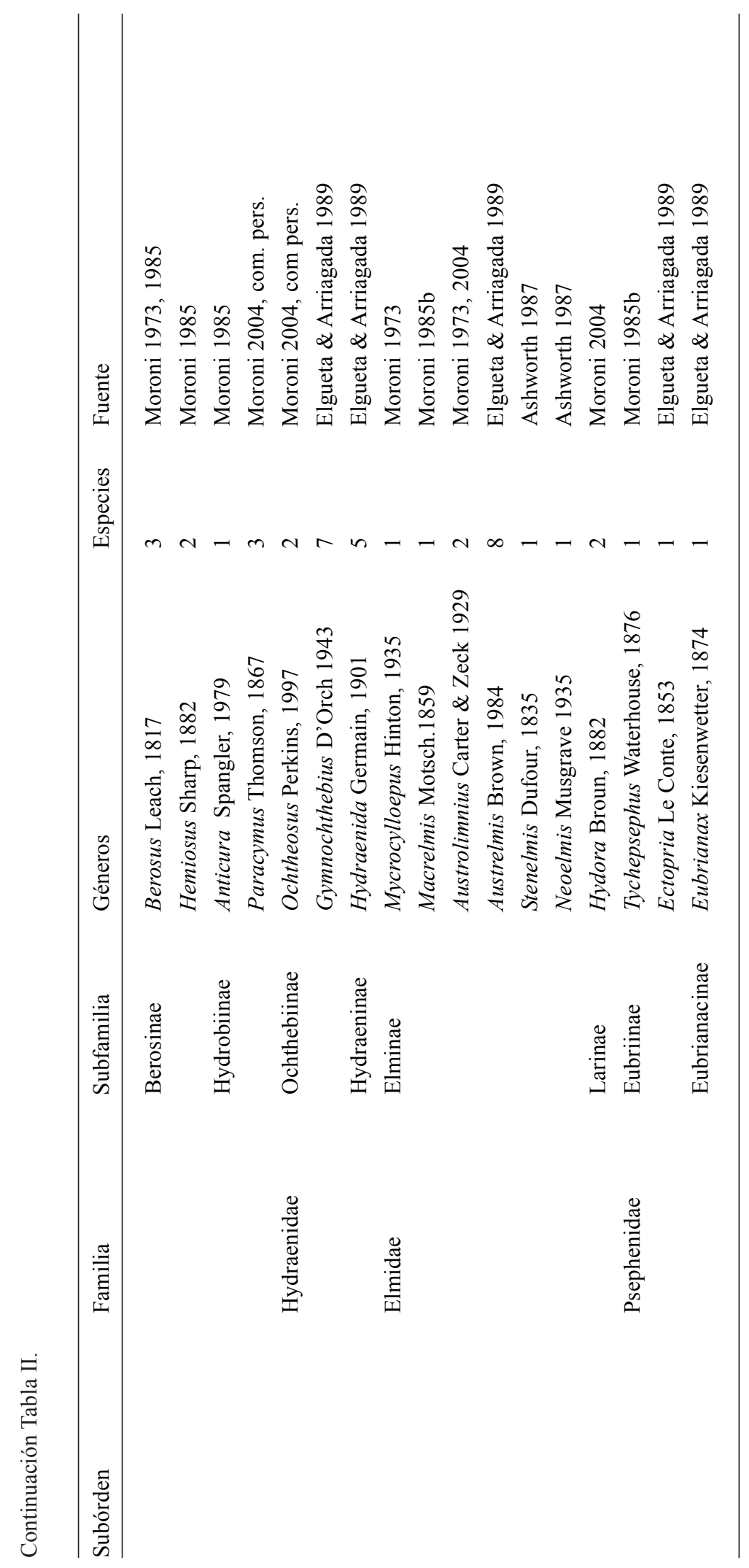




\section{Endemismo en Chile}

Territorios continentales: En base a los registros de localidades, existen algunos taxa que tienen distribuciones geográficas restringidas, situación que daría lugar a considerar un cierto grado de endemismo. Es el caso de Enochrus concepcionensis Moroni 2000 (Hydrophilidae), considerada por el autor endémica de las provincias de Concepción y Bío-Bío, y para el Parque Nacional Puyehue, Laccornis copetaloides (Ashworth \& Hoganson 1987). Otras especies son: Rhantus obscuricollis (se conoce solamente de Aysén en Chile y Neuquén en Argentina), Platynectes magellanicus (descrita sólo para la provincia de Magallanes); Rhantus antarcticus (solamente en la provincia de Concepción y Cautín); Lancetes flavipes (de la provincia de Magallanes en Punta Arenas); Lancetes towianicus (del lago Towia, Tierra del Fuego) y Lancetes kuscheli (provincia de Antofagasta). Para el caso de Elmidae, esta situación se presenta en las siguientes especies: Microcylloepus chilensis (ha sido descrita sólo en la provincia de Tarapacá en la Quebrada de Camarones); Austrelmis chilensis, A. trivialis, A. scissicollis y $A$. nyctelioides (descritas sólo para la provincia de Quillota) y Austrelmis elegans y A. costulata (de la provincia de Antofagasta, en Tumbre).

Territorios insulares: Según Guignot (1952), en el Archipiélago de Juan Fernández la fauna de Dytiscidae estaría compuesta solamente por tres especies, todas de la subfamilia Colymbetinae. De éstas, Lancetes bäckstromi y Anisomeria bistriata son endémicas para la isla Masafuera y Masatierra, respectivamente. Esta poca diversidad sería similar a lo que ocurre en las faunas insulares del océano Pacífico. Para Isla de Pascua se ha descrito como endémica a Bidessus skottsbergi (Moroni 1973). Para Isla Mocha fue descrita Rhantus signatus (Dytiscidae) por Reiche (1903); sin embargo esta especie se encuentra presente desde Antofagasta hasta Magallanes (Moroni, com. pers.).

\section{ACTUALIZACIÓN EN CONOCIMIENTO BIOLÓGICO}

El conocimiento sistemático de los coleópteros acuáticos es mayor que el biológico; la gran parte de la información disponible para las familias es muy general y está referida habitualmente a aspectos conocidos para fauna foránea o bien extrapolada a partir de las descripciones de algunos estados larvarios. Sólo tres trabajos han sido publicados referentes a estados ontogenéticos de especies chilenas y que corresponden a los aportes de Artigas (1963) para Psephenidae, Cekalovic (1974) para Dytiscidae y Moroni (1989) para Haliplidae. En la Tabla III se indican los roles tróficos que cumplen, en los ecosistemas de aguas continentales, las siete familias de coleópteros acuáticos consideradas en este trabajo.

\section{AsPeCtos ECOLÓGICOS}

Los aspectos biológicos y ecológicos de esta fauna, para la mayoría de las especies de coleópteros acuáticos, son desconocidos. A continuación se presentan comentarios por familia:

GYRINIDAE: Larvas y adultos son de hábitos acuáticos y depredadores; frecuentan cuerpos de agua de poca corriente. Se les ha recolectado como parte de fauna de deriva (Figueroa et al. 2003).

HALIPLIDAE: Su hábitat lo constituyen cuerpos de agua lénticos, con abundantes algas filamentosas y plantas subacuáticas, y fondos ricos en detritus. Larvas no nadadoras y fragmentadoras de régimen fitófago, se alimentan de algas filamentosas y viven entre el detritus. Los adultos se desplazan por el agua por movimientos alternados de las patas mesotorácicas. Poblaciones poco numerosas (Moroni 1980, Elgueta \& Arriagada 1989). Los huevos son puestos en el interior de tallos y hojas, en una cavidad que realizan con el ovipositor o mandíbulas o reunidos en ootecas adheridas a las plantas subacuáticas; la pupación ocurre fuera del agua, al interior de una cámara.

DYTISCIDAE: Presentes en la totalidad de aguas continentales de ambientes lénticos: lagos, lagunas, humedales y en cuerpos de agua permanentes y temporales. También existen especies adaptadas a condiciones extremas y en sectores de potamón. En zonas lóticas pueden estar presentes en zonas de desborde de poca profundidad. Larvas y adultos de hábitos acuáticos y carnívoros; los adultos presentan gran capacidad de vuelo. Distribución en todo el territorio y en la zona central llegan hasta 2.300 msnm. También se encuentran en salares del norte de Chile, en cuerpos de aguas salobres o ricas en algas amarillas que le dan el color amarillento al cuerpo de larvas y adultos.

Hydrophilidae: Adultos saprófagos y larvas depredadoras con digestión preoral; de hábitos acuáticos o semiacuáticos. Tropisternus setiger ha sido señalada como depredadora de Culicidae, aunque también realizan canibalismo entre ellas (Moroni, 
com. pers.). Para esta misma especie se han realizado algunos estudios con fauna parásita asociada, como son los trabajos de Pacho (1996), relacionado con ácaros acuáticos, y Murillo et al. (2000), quienes dan a conocer la presencia de nemátodos Mermithidae en adultos de T. setiger. En Tropisternus, los huevos son protegidos por ootecas adheridas a las plantas subacuáticas. Los adultos de la subfamilia Sphaeridiinae son los únicos que presentan hábitos terrestres. Estos insectos al parecer viven en todo tipo de ambientes e incluso una especie del género Berosus ha sido señalada en aguas termales en Brasil (Oliva 1992).

HYDRAENIDAE: Insectos pequeños, no nadadores, por lo cual se desplazan caminando sobre rocas y algas en las riberas de cuerpos de agua, o en los márgenes de corrientes de aguas claras y fondo arenoso ( Perkins 1980).

Elmidae: Acuáticos en estado adulto y larvario, se encuentran generalmente en fondos arenosos, gravosos o sumergidos entre la vegetación. Se alimentan de algas y detritus, y también de microorganismos y pequeños invertebrados acuáticos. Todos los adultos presentan un plastrón ventral con pelos hidrófugos. Esta familia ha sido reportada como parte de la fauna de deriva en zonas de ritrón del río Rucué (tributario del lago Laja), también del estero Nonguén (Araya 2000) y en el río Damas (Figueroa 1999, Figueroa et al. 2003).

PSEPHENIDAE: Larvas de hábitos acuáticos, se encuentran adheridas al sustrato y son de aspecto crustaceiformes; se encuentran en zonas de ritrón que corresponden a sectores de alta pendiente, con altas velocidades de corrientes, temperaturas bajas y estables y altas concentraciones de oxígeno. En relación con estados ontogenéticos, Artigas (1963) describió la larva de Tychepsephus felix, cuyo hábitat es aguas corrientes y cristalinas, con bajo contenido orgánico y poca profundidad.

\section{USO COMO BIOINDICADORES}

En el manejo de ecosistemas acuáticos, los coleópteros han probado ser un importante grupo taxonómico que puede ser biomonitoreado. Debido a su diversidad en número de especies, variación en tamaño y condiciones ecológicas, representan un grupo ideal para estudios de impacto ambiental, propuestas de conservación y estudios de biodiversidad en un sentido amplio. Entre los macroinvertebrados bentónicos, los
Elmidae serían particularmente sensibles a la contaminación (Figueroa et al. 2003).

\section{COLECCIONES DE REFERENCIA}

En el país existen unas pocas colecciones de coleópteros acuáticos que son detalladas a continuación:

a) Museo Nacional de Historia Natural (MNHN), Santiago: Esta colección data del tiempo en que Philibert Germain trabajó en esta institución entre 1893 y 1911, que incluye material recolectado por el mismo, principalmente en la zona central del país. En la actualidad, la colección incluye material tipo de Haliplidae, Dytiscidae, Hydrophilidae, Hydraenidae y Elmidae. Camousseight y Moroni (1976) publicaron el listado de los tipos depositados en esta colección.

b) Museo de Zoología de la Universidad de Concepción (UCCC), Concepción: Cuenta con una importante colección de referencia de géneros y especies de las familias Gyrinidae (Andogyrus ellipticus, A. seriatopunctatus); Haliplidae (Haliplus fuscipennis); Dytiscidae (Laccophilus chilensis, L. ivietae, Laccornellus copetaloides, Lancetes debilis, L. flavosculatus, L. nigriceps, L. praemosus, L. tarsalis, Leuronectes gaudichaudi, Liodesssus delfini, Megadytes australis, Rhantus antarcticus, $R$. signatus, $R$. validus); Hydrophilidae (Berossus bruchianus, B. chalcocephalus, B. pallipes, Cylorygmus lineatopunctatus, Enochrus claudei, E. fulvipes, E. vicinus, Hemiosus dejeani); Hydraenidae Hydrochus stolpi, Tropisternus setiger; Elmidae (Elmis chilensis, E. ovalis) y Psephenidae (Tychepsephus felix).

c) Instituto de Entomología. Universidad Metropolitana de Ciencias de la Educación, Santiago: Esta colección data de 1960 y cuenta con ejemplares de Dytiscidae (Lancetes nigriceps, Leuronectes gaudichaudi, Rhantus signatus y Lacornis copetaloides); Gyrinidae (Andogyrus ellipticus); Hydraenidae (Gymnochthebius germaini) e Hydrophilidae (Tropisternus setiger, Enochrus sp.). d) Particulares: Juan Carlos Moroni posee una colección que alcanza aproximadamente a 181 especies y alrededor de 3.000 ejemplares. La colección incluye algunos paratipos y material exclusivo de la fauna de Argentina, no presentes en otra colección chilena. 
Coleópteros dulceacuícolas de Chile: Jerez, V. \& J. MoronI

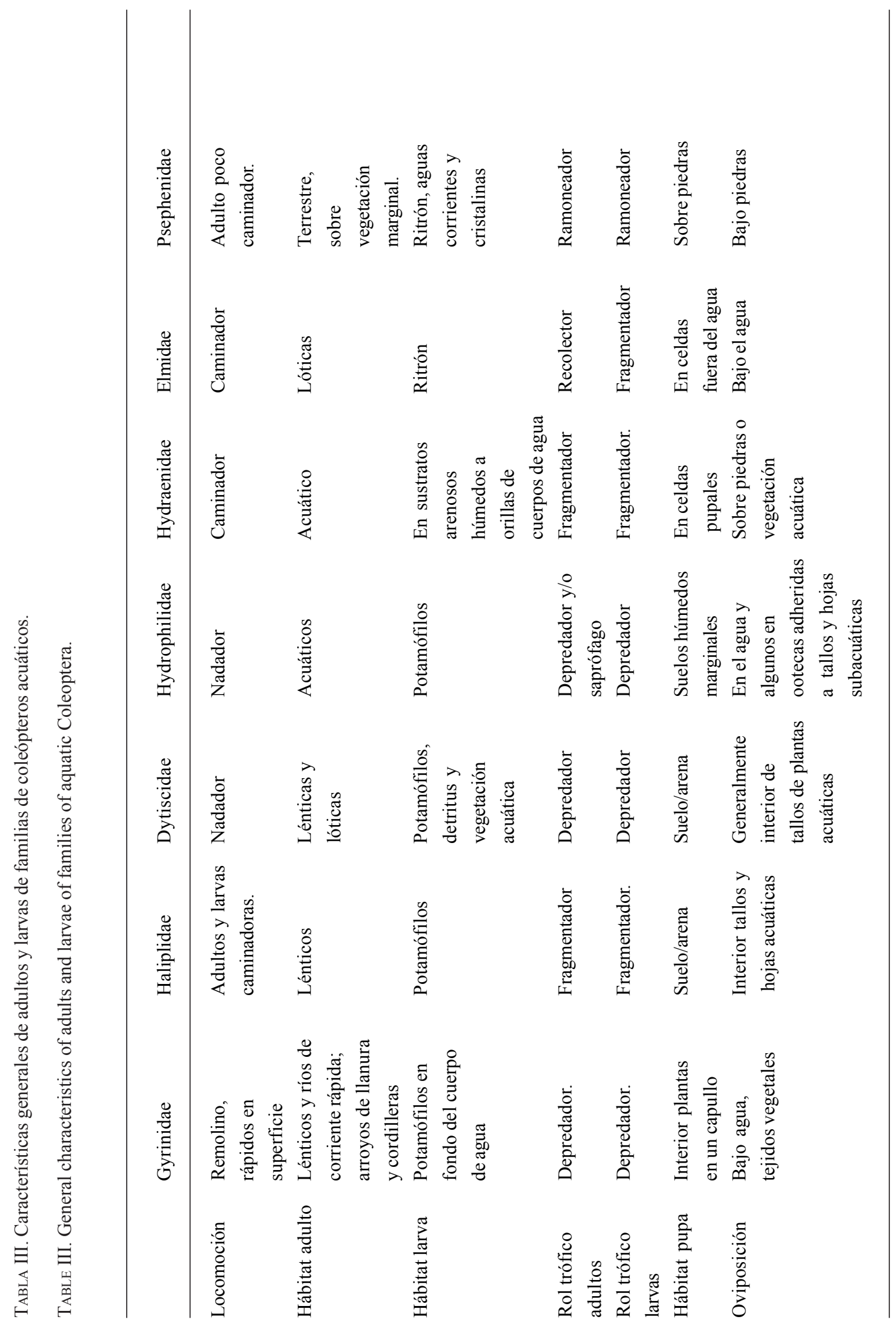




\section{AGRADECIMIENTOS}

Los autores agradecen a la Dirección de Investigación de la Universidad de Concepción por el financiamiento con aportes del Proyecto DIUC 203.113.061-1.0. A Minerva Contreras y Elvira Solar por datos de la colección del Museo de Zoología de la Universidad de Concepción, a Jaime Solervicens por información de la colección del Instituto de Entomología, Universidad Metropolitana de Ciencias de la Educación. Finalmente a Ariel Camousseigh, Mario Elgueta y Fresia Rojas de la sección Entomología del Museo Nacional de Historia Natural, Santiago.

\section{BIBLIOGRAFIA}

Araya, E.M. 2000. Colonización de sustratos artificiales por macroinvertebrados bentónicos en un ecosistema fluvial de baja intervención antrópica, Estero Nonguén (VIII Región, Chile). Tesis de Magíster en Zoología. Facultad de Ciencias Naturales y Oceanográficas. Universidad de Concepción. 128 pp.

Arias, E. 2000. Coleópteros de Chile. Fototeknika. Santiago de Chile. 209 pp.

Artigas, J. 1963. Descripción de la larva de un Psefenido chileno (Coleoptera-Psephenidae). Gayana Zoología 8: 3-9.

Ashworth, A.C. \& J.W. Hoganson. 1987. Coleoptera bioassociations along an elevational gradient in the Lake Region of Southern Chile, and comments on the postglacial development of the fauna. Annals of the Entomological Society of America 80(6): 865-895.

Camousseight, A. \& J. Moroni. 1976. Los tipos de coleópteros acuáticos depositados en la colección del Museo Nacional de Historia Natural de Chile (MNHN). Noticiario Mensual, Museo Nacional de Historia Natural. 21(242-243): 3-6.

Cekalovic, T. 1974. Descripción de la larva de Megadytes australis (Germain), 1854 (Coleoptera, Dytiscidae). Boletín de la Sociedad de Biología de Concepción, Chile 48: 35-40.

Elgueta, M. \& G. Arriagada. 1989. Estado actual del conocimiento de los coleópteros de Chile (Insecta: Coleoptera). Revista Chilena Entomología 17: 5-60.

Elgueta, M. 2000. Coleópteros de Chile. En: Hacia un proyecto SYTED para el inventario y estimación de diversidad entomológica en Iberoamerica (Eds. F. Martin-Piero, J.J. Morrone \& A. Melick), pp. 145-154. Pribes Sea, Zaragoza.

Fernández, L.A. \& E. A. Domizi. 1983. Estudio preliminar sobre la biología de Tropisternus (Pristoternus) setiger Germar (Coleoptera, Hydrophilidae). Revista de la Sociedad
Entomológica Argentina 42 (1-4) : 261-265.

FigueroA, R. 1999. Macroinvertebrados bentónicos como indicadores biológicos de calidad de agua: Río Damas, Osorno (X Región, Chile). Tesis Magíster en Ciencias mención Zoología. Facultad de Ciencias Naturales y Oceanográficas. Universidad de Concepción. 105 pp.

Figueroa, R., C. Valdovinos, E. Araya \& O. Parra. 2003. Macroinvertebrados bentónicos como indicadores de calidad de agua de ríos del sur de Chile. Revista Chilena de Historia Natural 76 (2): 275-285.

Germain, P. 1911. Catálogo de los coleópteros chilenos del museo nacional. Boletín del Museo Nacional de Chile. 3(1) : 47-73.

Guignot, F. 1952. Los insectos de la isla Juan Fernández. 10. Dytiscidae (Coleoptera). Revista Chilena de Entomología 2: 113-115.

Hinton, H.E. 1965. A revision of the australian species of Austrolimnius (Coleoptera: Elmidae). Australian Journal of Zoology 13(1): 97 - 172

Lawrence, J. F. \& A. F. Newton. 1995. Families and subfamilies of Coleoptera (with selected genera, notes, references and data on family-group names). En: Biology, Phylogeny and Classification of Coleoptera: Papers celebrating the $80^{\text {th }}$ birthday of Roy A. Crowson (Eds. J. Pakaluk \& S.A. Slipinski), pp. 799-909. Muzeum I Instytut Zoologi PAN, Warszawa.

Moroni, J. 1973. Elenco sistemático, sinonímico y distribución de coleópteros acuáticos chilenos. Revista Chilena de Entomología 7: 193-206.

Moroni, J. 1980. Aporte al conocimiento de los Haliplidae de Chile (Coleoptera: Dytiscoidea). Revista Chilena de Entomología 10: 29-33.

Moroni, J. 1985a. Redescripción de Cylorygmus lineatopunctatus D'Orchymont, 1933 (Coleoptera: Hydrophilidae: Rygmodini). Revista Chilena de Entomología 12: 145-151.

Moroni, J. 1985b. Addenda y corrigenda al elenco sistemático, sinonímico y distribución de coleópteros acuáticos chilenos. Revista Chilena de Entomología 12: $169-175$

Moroni, J. 1988. Revisión del género Rhantus Dejean en Chile (Coleoptera: Dytiscidae: Colymbetinae). Revista Chilena de Entomología 16: 49-64.

Moroni, J. 1989. Aporte al conocimiento de los Haliplidae de Chile. Descripción del tercer instar larval de Haliplus valdiviensis Moroni, 1980 (Coleoptera:Dytiscoidea). Revista Chilena de Entomología 17: 89-95.

Oliva, A.1992. Nuevas especies sudamericanas del genero Berosus (Coleoptera; Hydrophilidae). Revista de la Sociedad Entomológica Argentina 51 (1 - 4): 87-95.

PACHO, R.R. 1996. Acaros acuáticos asociados a Tropisternus (Pristoternus) setiger Germar, 1824 (Col. Hydrophilidae) en Chile. Tesis Magíster en Ciencias mención Zoología. Facultad de Ciencias Naturales y Oceanográficas. Universidad de Concepción. 144 pp. 
Coleópteros dulceacuícolas de Chile: Jerez, V. \& J. Moroni

Perkins, P.D. 1980. Aquatic beetles of the Family Hydraenidae in the Western Hemisphere: Classification, Biogeography and inferred Phylogeny (Insecta: Coleoptera). Quaestiones Entomologicae 16: 1-554.

Philippi, F. 1887. Catálogo de los coleópteros de Chile. Anales de la Universidad de Chile 71: 1- 190.

Ribera, I. 2000. Notes on the genus Hydraenida Germain, with description of a new species from Chile (Coleoptera:Hydraenidae). Coleopterological Review 70: 53-66.

Ribera, I., J.E. Hogan \& A.P. Vogler. 2002. Phylogeny of Hydradephagan water beetles inferred from 185 rRNA sequences. Molecular Phylogenetics and Evolution 23(1) 43-62.

Solervicens, J. 1995. Consideraciones generales sobre los insectos, el estado de su conocimiento y las colecciones. En J.Simonetti, M.T.K.Arroyo, A.E. Spotorno y E.Lozada. (eds.). Diversidad Biológica de Chile: 198 210. Conicyt. Chile.

Trémouilles, E.R., A. Oliva \& A.O. Bachmenn. 1995. Insecta Coleoptera. En: Ecosistemas de aguas continentales: Metodologías para su estudio (Eds. E.C. Lopretto \& G.Tell), pp. 1133-1197. Ediciones Sur, Buenos Aires.

Fecha de recepción: 25.08.05

Fecha de aceptación: 12.12.05 